\title{
Gelatin Tannate for Acute Childhood Gastroenteritis: A Randomized, Single-Blind Controlled Trial
}

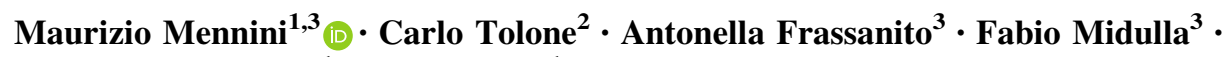 \\ Salvatore Cucchiara ${ }^{1} \cdot$ Marina Aloi $^{1}$
}

C) Springer International Publishing Switzerland 2016

\begin{abstract}
Background Oral rehydration therapy is the recommended treatment for acute childhood gastroenteritis. The aim of this study was to assess the efficacy and safety of gelatin tannate plus oral rehydration compared with oral rehydration alone.

Methods We conducted a multicenter, parallel, randomized, controlled, single-blind, prospective, open-label trial. A central randomization center used computer generated tables to allocate treatments. The study was performed in two medical centers in Italy. Sixty patients 3-72 months of
\end{abstract}

Electronic supplementary material The online version of this article (doi:10.1007/s40272-016-0207-z) contains supplementary material, which is available to authorized users.

Maurizio Mennini

Maurizio.mennini@gmail.com

Carlo Tolone

carlo.tolone@unina2.it

Antonella Frassanito

antonella.frassanito@uniroma1.it

Fabio Midulla

fabio.midulla@uniroma1.it

Salvatore Cucchiara

salvatore.cucchiara@uniroma1.it

Marina Aloi

marina.aloi@uniroma1.it

Pediatric Gastroenterology and Liver Unit, Sapienza University of Rome, Viale Regina Elena 324, 00161 Rome, Italy

2 Department of Pediatrics, Second University of Naples, Viale Beneduce 10, 81100 Caserta, Italy

3 Department of Pediatrics, Sapienza University of Rome, Viale Regina Elena 324, 00161 Rome, Italy age with acute gastroenteritis were recruited (median age 18 months; age range 3-66 months): 29 received an oral rehydration solution (ORS) and 31 an ORS plus gelatin tannate (ORS $+\mathrm{G})$. The primary outcome was the number of bowel movements 48 and $72 \mathrm{~h}$ after initiating treatment. Secondary outcomes were: duration of diarrhea, stool characteristics and adverse events.

Results No patient was lost at follow-up. No significant difference in the number of bowel movements after $48 \mathrm{~h}$ was reported $(2.7 \pm 1.3$ ORS $+\mathrm{G} ; 3.2 \pm 0.8$ ORS; $p=0.06)$, although the ORS $+\mathrm{G}$ group showed a significant improvement in stool consistency ( $3.7 \pm 1.0$ vs. $4.3 \pm 0.8 ; p=0.005)$. At $72 \mathrm{~h}$, a significant reduction in bowel movements was reported in the ORS $+\mathrm{G}$ group compared with the ORS group $(1.0 \pm 1.4$ vs. $2.0 \pm 1.7$; $p=0.01)$. Mean duration of diarrhea was significantly lower in the ORS $+\mathrm{G}$ group than in the ORS only group $(76.8 \pm 19.2$ vs. $108 \pm 24.0 \mathrm{~h} ; p<0.0001)$. No adverse events were reported.

Conclusions Gelatin tannate added to oral rehydration in children with acute diarrhea was associated with a significant decrease in bowel movements at $72 \mathrm{~h}$, with an early improvement in the stool consistency and shorter disease duration.

Clinical Trial Registration NCT02644200-Gelatin Tannate as Treatment for Acute Childhood Gastroenteritis (https://www.clinicaltrials.gov). 


\section{Key Points}

Oral rehydration therapy is the main treatment recommended for acute gastroenteritis. Recently, gelatin tannate has been proposed as a theoretically effective new treatment. Clinical data on its efficacy are very limited.

This is the first single-blind, prospective, randomized, parallel study in children that demonstrates that gelatin tannate can be effective, well-tolerated, and safe in addition to oral rehydration therapy.

\section{Background}

Acute gastroenteritis is one of the most common diseases in childhood, with up to 3-5 billion cases of acute gastroenteritis and nearly 2 million deaths per year in children under 5 years. It is also a significant healthcare burden both in developing and developed countries [1]. According to the European Society for Paediatric Gastroenterology, Hepatology and Nutrition (ESPGHAN) guidelines for the treatment of acute gastroenteritis, oral rehydration with hypo-osmolar solutions is the mainstay of therapy [2]. However, although rehydration therapy has achieved a significant reduction in mortality and morbidity due to dehydration, it does not affect the duration of diarrhea $[3,4]$. Therefore, active treatments that may impact on the severity of diarrhea have been implemented, including specific probiotics, such as Lactobacillus GG or Saccharomyces boulardii or drugs such as racecadotril or diosmectite [5-7].

Recently, gelatin tannate, a compound based on tannic acid suspension in a gelatin solution, has been proposed as a theoretically effective treatment for pediatric acute gastroenteritis [8]. Tannins are mostly water-soluble phenolic compounds, and are well-known antioxidants sourced from medicinal plants, foods, and edible fruits. Vegetable tannins have attracted a lot of attention in recent years because of their multifunctional properties, beneficial to human health, demonstrated through in vitro and in vivo assays, and recently, an antidiarrhoic effect of gelatin tannate has been reported [9, 10]. Scaldaferri et al. evaluated the therapeutic effect and mechanisms of action of gelatin tannate using the dextran sulfate sodium (DSS)-induced acute colitis mouse model. Gelatin tannate not only significantly improved disease severity in terms of disease activity index (DAI), but also body weight and histological score [11].
Furthermore, gelatin tannate has been reported to modulate the composition of gut microbiota, restore mucus layer, with an improvement of gut permeability, and exert a direct anti-inflammatory effect in the gastrointestinal mucosa [12, 13]. Clinical data on the efficacy of gelatin tannate in acute gastroenteritis are very limited. In 2009, Esteban Carretero et al. reported a significant reduction in the number of loose stools in 97 children treated with oral rehydration solution (ORS) plus gelatin tannate $(\mathrm{ORS}+\mathrm{G})$, compared with 114 treated with ORS alone in a non-randomized, non-blinded clinical study [10]. No drug-related adverse events were reported. More recently, a randomized, blinded, placebo-controlled trial was performed in 40 adults with acute diarrhea [14]. A significant reduction in the number of loose stools was reported in the active group compared with placebo. Based on these two studies, a systematic review on the efficacy of gelatin tannate in acute gastroenteritis published in 2014 concluded that there is not enough evidence to support the use of gelatin tannate in clinical practice [15].

The aim of this study was to evaluate the efficacy and safety of gelatin tannate combined with ORS compared with ORS in children with acute gastroenteritis.

\section{Methods}

This was a single-blind, prospective, randomized, parallel study conducted in two pediatric services of two tertiarycare hospitals.

Children were eligible for the study if they were 3-72 months of age with acute gastroenteritis, as defined by having at least three loose stools within the previous $24 \mathrm{~h}$ and/or a change in stool consistency to loose or liquid according to the modified Bristol Stool Form Scale for Children (m-BSFS-C) lasting for no longer than 3 days $[16,17]$. Patients with gastroenteritis for more than 3 days, those with chronic gastrointestinal diseases, or those receiving other antidiarrheal drugs within 2 weeks prior to enrollment (i.e., antibiotics, probiotics, salicylates, loperamide, racecadotril, diosmectite) were excluded from the study.

All children were randomly assigned to the groups using a computer-generated randomization list. The sequence was concealed and envelopes for consecutive patients prepared accordingly. As each patient was recruited, the next envelope in sequence was opened to determine the allocation of the patient. Group 1 received ORS (controls) and group 2 received ORS plus gelatin tannate (Gelenterum sachet $250 \mathrm{mg}$, manufactured by Novintethical Pharma Sagl, Lugano, Switzerland, marketed by ACRAF S.p.A Italy) as follows: $250 \mathrm{mg}$ every $6 \mathrm{~h}$ if weighing less than $15 \mathrm{~kg}$ and $500 \mathrm{mg}$ every $6 \mathrm{~h}$ if weighing over $15 \mathrm{~kg}$, 
for 5 days. Allocation and management of enrolled patients were performed by two physicians (M.M. and C.T.), while the study coordinator (M.A.) was blinded to the group allocation and the treatment received until the completion of the trial.

Patients in both treatment groups received a standard rehydration solution, following the recommendations of the ESPGHAN [2].

During the initial visit, the following data were collected: demographic variables, family history of gastrointestinal disease, patient's medical history and characteristics of the current gastroenteritis. Physical examination was performed, collecting data on weight, body temperature, heart and respiration rates, blood pressure, degree of dehydration, and any additional tests required for clinical purposes by the treating physician. Dehydration was evaluated using the Clinical Dehydration Scale (CDS), assessing four items: general appearance, eyes, mucous membranes, and tears [18]. Parents received a symptom diary (see electronic supplementary material, online resource 1) and were instructed to complete it, assessing the number of stools, stool consistency according to $\mathrm{m}$-BSFS-C, food and liquid intake, adverse events, and need for other therapies during the study period. All parents were also instructed to determine the daily number of stools. During the 24-, 48-, and 72-h evaluation, tolerance to treatment, side effects, changes in concomitant medication, need for additional visits to the pediatrician or emergency room, as well as data regarding the outcome of the gastroenteritis (number of bowel movements per day and consistency, vomiting, and weight) were monitored. The duration of diarrhea was defined as the time in hours from enrollment to the last abnormal (loose or liquid) stool. Last abnormal stool was defined when the child passed normal stool or no stool for the next $24 \mathrm{~h}$. Stool consistency was evaluated on m-BSFS-C and defined as follows: (1) separate hard lumps, like nuts; (2) sausage-shaped but lumpy; (3) like a sausage or snake, smooth and soft; (4) fluffy pieces with ragged edges, a mushy stool; and (5) watery, no solid pieces [16]. Therapeutic adherence was evaluated on the basis of the number of doses received in $48 \mathrm{~h}$ of treatment; good compliance was defined as when the patients received $75 \%$ or more of doses according to the patient's bodyweight.

At the last visit, on day 7, symptom diary, including side effect occurrence, was evaluated.

The main objective of this study was to determine the efficacy of treatment according to the number of bowel movements after 48 and $72 \mathrm{~h}$ after initiation of therapy with ORS $+\mathrm{G}$ versus ORS alone. Secondary aims were to evaluate differences in the length of the diarrhea, stool characteristics, and the safety and tolerance to the study drug.
The study protocol was defined in accordance with the Declaration of Helsinki, and was approved by the ethical committee of each participating center (593/12). Written informed consent was obtained from parents of all children.

\subsection{Statistical Analysis}

All data were summarized and displayed as mean \pm standard deviation (SD) for the continuous variables. Categorical data were expressed as frequencies and percentages. Comparison of groups was performed using Student's $t$ test for unpaired data in a two-group comparison and one-way analysis of variance (ANOVA) with Bonferroni's test for multiple group comparisons. Chisquare tests with Fisher's correction were used to address any differences for categorical variables, as needed. A $p$ value of 0.05 or less was considered as significant. Assessment of the duration of diarrhea and specific variables was performed using Pearson and Spearman rank correlation. Kaplan-Meier survival analysis was used to estimate the survival distribution of the duration of diarrhea in the two groups. GraphPad statistical package was used to perform all statistical analyses (GraphPad Software, Inc.).

\section{Results}

Ninety-six children (42 males; median age 19.6 months, age range 3-69 months) were initially recruited from June 2012 to June 2014: three were excluded because of concomitant chronic diseases; 19 were already receiving racecadotril; one started antibiotic therapy; two who received ORS needed intravenous rehydration; and 11 refused to take part in the study. Therefore, 60 children [30 males (50\%); median age 18 months, age range 3-66 months] were included in the study (Fig. 1).

Patients were randomized as follows: 29 (median age 18 months; range 7-43 months) received an ORS; 31 children (median age 20 months; range 3-66 months) received ORS $+\mathrm{G}$.

Both groups were similar in terms of baseline clinical characteristics: median age, gender distribution, weight, concomitant symptoms, and CDS (Table 1). Seventeen patients in group 1 and 14 in group 2 self-managed their toileting.

The number of bowel movements at enrollment was $5.1 \pm 2.5$ in the ORS group and $6.2 \pm 2.9$ in the ORS $+\mathrm{G}$ group $(p=0.11)$.

At 24 and $48 \mathrm{~h}$, the number of bowel movements was not significantly different between the two groups $(3.0 \pm 1.1 \mathrm{ORS}$ vs. $2.9 \pm 1.0 \mathrm{ORS}+\mathrm{G} ; p=0.81$ and $3.2 \pm 0.8$ vs. $2.7 \pm 1.3 ; p=0.06$, respectively). However, at $72 \mathrm{~h}$, children with ORS $+\mathrm{G}$ had a significant reduction 
Fig. 1 Consort flow diagram of the two groups' progress through the phases of the parallel randomized trial: enrollment, intervention allocation, follow-up, and data analysis

Table 1 Baseline clinical characteristics of the children enrolled in the study

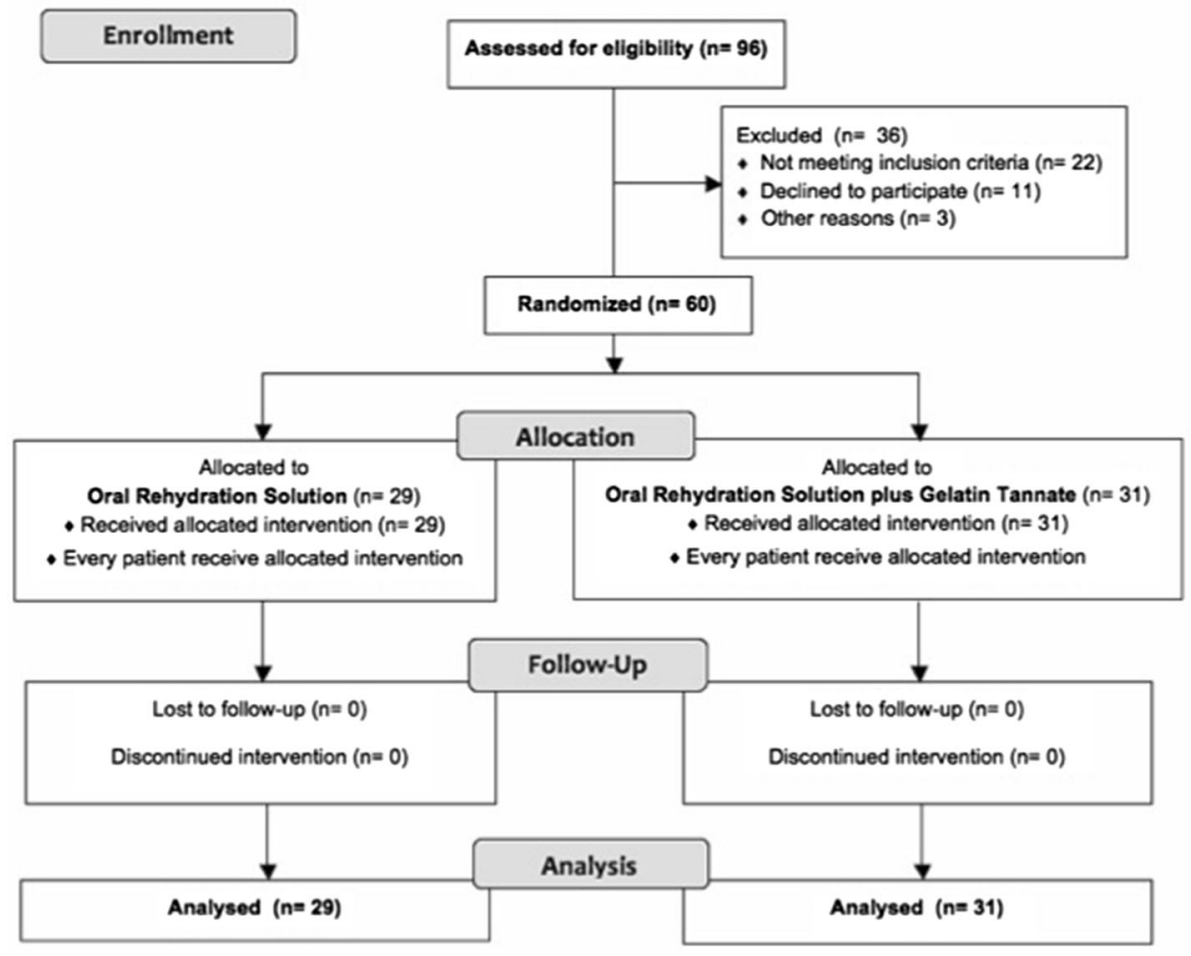

\begin{tabular}{llll}
\hline & ORS group $(N=29)$ & ORS + G group $(N=31)$ & $p$ \\
\hline Median age (range), months & $18(7-43)$ & $20(3-66)$ & 0.14 \\
Male (\%) & $15(52)$ & $15(48)$ & 1.0 \\
Mean weight (kg $\pm \mathrm{SD})$ & $10.77 \pm 2.31$ & $12.02 \pm 3.27$ & 0.09 \\
Mean blood pressure (mmHg) & Systolic: $102.3 \pm 6.97$ & Systolic: $104.5 \pm 9.02$ & 0.86 \\
& Diastolic: $58.9 \pm 3.14$ & Diastolic: $62.5 \pm 5.22$ & 0.77 \\
Duration of diarrhea before enrollment (h) & 79.9 & 55.0 & 0.12 \\
Bowel movements/day & 5.13 & 6.29 & 0.11 \\
Abdominal pain (\%) & $8(28)$ & $15(48)$ & 0.11 \\
Vomiting (\%) & $21(72)$ & $16(52)$ & 0.11 \\
Fever (\%) & $18(62)$ & $14(45)$ & 0.20 \\
Mean Clinical Dehydration Scale & 3.10 & 3.26 & 1.0 \\
Dehydration (\%) & Mild: $3(10)$ & Mild: $2(6)$ & 0.47 \\
& Moderate: $25(86)$ & Moderate: $26(84)$ & 0.35 \\
\hline
\end{tabular}

ORS oral rehydration solution, $O R S+G$ oral rehydration solution plus gelatin tannate, $S D$ standard deviation in bowel movements $(1.0 \pm 1.4)$ compared with those treated with ORS $(2.0 \pm 1.8 ; p=0.01)$ (Fig. 2). At 48 h, a significant improvement in bowel consistency, according to m-BSFS-C, was reported in the ORS + G group compared with the ORS group $(3.7 \pm 1.0$ and $4.3 \pm 0.8 ; p=0.005)$.

The mean duration of diarrhea was significantly shorter in patients treated with ORS $+\mathrm{G}$ than in those treated with ORS only $(76.8 \pm 19.2$ vs. $108 \pm 24.0 \mathrm{~h} ; p<0.0001)$, with a mean difference of $31.2 \mathrm{~h}$. Figure 3 shows the time to diarrhea resolution in the two groups (log-rank 5.7; $p=0.01)$. In a univariate analysis of baseline clinical variables related to the duration of diarrhea at $72 \mathrm{~h}$, we found age to be the only factor correlated $[r=0.26 ; 95 \%$ confidence interval (CI) $0.01-0.48 ; p=0.03$ ] (Table 2). After $72 \mathrm{~h}$ of treatment, 28 patients $(96.5 \%)$ in the ORS group and $29(93.5 \%)$ in the ORS $+\mathrm{G}$ group did not complain of any symptoms: one child $(3 \%)$ in the ORS group and one (3\%) in the ORS $+\mathrm{G}$ complained of 


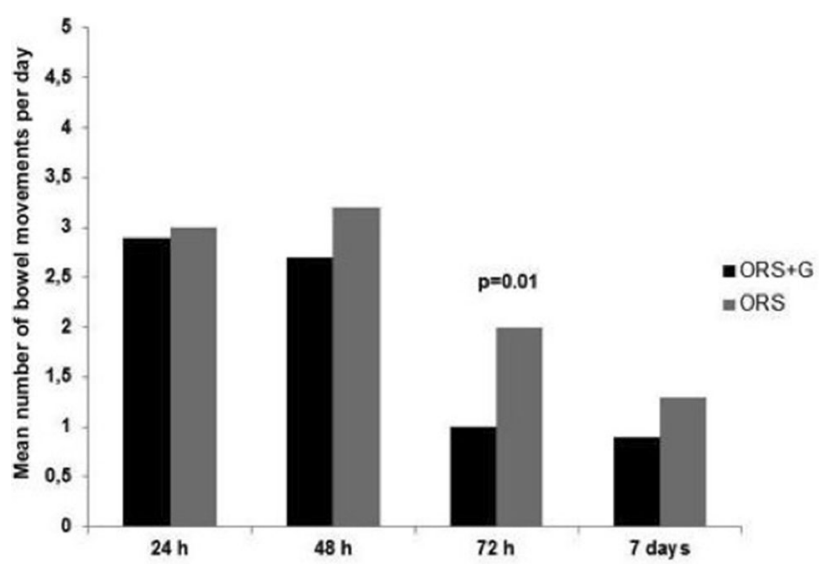

Fig. 2 Mean of bowel movements per day. ORS $+\mathrm{G}$ children had a significant reduction in the bowel movements compared with those treated with ORS only at $72 \mathrm{~h}$. ORS oral rehydration solution, $O R S+G$ oral rehydration solution plus gelatin tannate

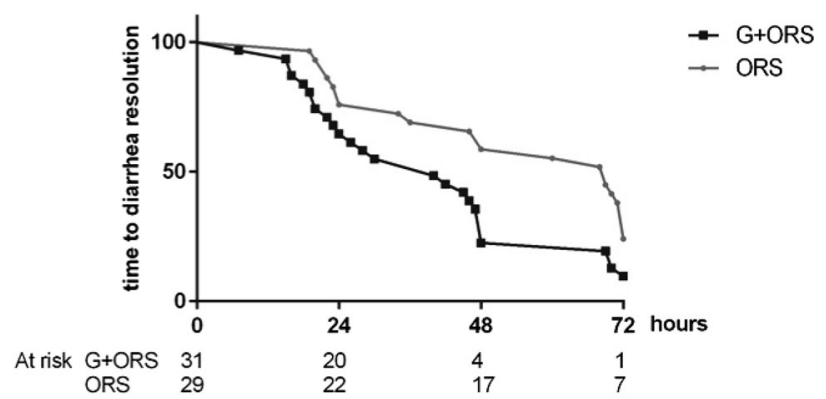

Fig. 3 Kaplan-Meier analysis. The Kaplan-Meier estimator shows the fraction of patients still affected by diarrhea after the start of treatment. ORS oral rehydration solution, $G+O R S$ oral rehydration solution plus gelatin tannate

abdominal pain, and one in the ORS + G group of vomiting. However, diarrhea was still present in seven patients of the ORS group $(24.1 \%)$ and in one patient of the ORS + G group (3.2\%).

Three patients in the ORS group and one in the ORS $+\mathrm{G}$ group needed additional visits to the primary doctor during treatment and one of the ORS group resorted to the emergency room; all had diarrhea lasting more than $72 \mathrm{~h}$.
All parents filled out the diary completely.

At 7-day follow-up, tolerability and drug acceptance were evaluated: all patients received $75 \%$ or more of the drug doses according to the patient's weight. One patient reported the occurrence of nausea about 20 min after administration of gelatin tannate. No other adverse events were reported.

\section{Discussion}

The most recent ESPGHAN guidelines for the treatment of acute gastroenteritis clearly state that oral rehydration with a hypo-osmolar solution is the main treatment and should be started as soon as possible [2].

Recent data have highlighted and introduced the possibility of using gelatin tannate, a mucosal barrier protector, as an innovative approach in the management of intestinal diseases, with the aim of enhancing mucus barrier activity and restoring the gut barrier [11]. Nevertheless, there are very limited data on its efficacy in children with acute diarrhea, although a randomized controlled trial performed in adults with acute gastroenteritis reported its good efficacy and safety in this setting [14].

The objective of our single-blind, prospective, randomized, parallel study was to compare the efficacy of combined therapy with gelatin tannate and oral rehydration with that of oral rehydration alone in children younger than 6 years affected with acute gastroenteritis as outpatient care.

We found patients treated with ORS $+G$ to have a significant decrease in the number of bowel movements after $72 \mathrm{~h}$ of treatment, when compared with those managed with ORS alone, while no significant difference was shown after 24 and $48 \mathrm{~h}$, and a significant improvement of bowel consistency was seen after $48 \mathrm{~h}$ of treatment.

Since it has already been demonstrated that gelatin tannate exerts its action by restoring the physiological function of the intestine, one may speculate that the drug is able to provoke a clinical effect only after restoring the intestinal barrier and thus will not be immediately effective $[9,11]$. Furthermore, the slow action may be also due to the well-
Table 2 Univariate analysis of baseline clinical factors related to the persistence of diarrhea after 3 days in 60 children with acute gastroenteritis

\begin{tabular}{lrll}
\hline & $r$ & $95 \%$ CI & $P$ values \\
\hline Age & 0.26 & 0.01 to 0.48 & 0.03 \\
Gender (male) & 0.06 & -0.19 to 0.31 & 0.60 \\
Symptom duration at the enrollment & 0.06 & -0.19 to 0.30 & 0.64 \\
Number of bowel movements & -0.02 & -0.27 to 0.23 & 0.85 \\
Vomiting & -0.15 & -0.39 to 0.1 & 0.23 \\
Abdominal pain & 0.20 & -0.05 to 0.43 & 0.11 \\
Fever & 0.11 & -0.14 to 0.36 & 0.37 \\
\hline
\end{tabular}

$C I$ confidence interval 
known astringent properties of tannins allowing the absorption of proinflammatory mucoproteins from the intestinal mucus and their elimination through the feces [19].

Overall, the mean duration of diarrhea was significantly shorter in patients treated with ORS $+\mathrm{G}$ than in those given ORS only, with a mean difference of $31.2 \mathrm{~h}$. These data are in keeping with the clinical effect of gelatin tannate in adults and with previous studies on the efficacy of other drugs suggested for the treatment of acute gastroenteritis [14]. Salazar-Lindo et al. reported a reduction in the duration of diarrhea in racecadotril-treated children up to $44 \mathrm{~h}$, compared with controls, while diosmectite is effective in reducing acute gastroenteritis by $23.39 \mathrm{~h}[7,20]$.

Compliance with administration was good; every patient received $75 \%$ or more of doses according to the patient's weight. This result confirms a good ease of administration. Safety was similar for the two treatment groups, with only one patient reporting the occurrence of nausea during a single administration of gelatin tannate. Three patients in the ORS group and one in the ORS $+\mathrm{G}$ group needed additional visits to the primary doctor during treatment, and one in the ORS group needed additional evaluation at the emergency room. These data suggest that duration of illness could be an important reason to utilize primary care or the emergency room.

We tried to identify predictive factors for prolonged diarrhea, and we found only age to be significantly correlated, with a duration of diarrhea greater than 3 days for older compared with younger children. Conversely, a previous study conducted in developing countries reported young age to be a risk factor for prolonged diarrhea [21]. This result could be related to the different study setting and design and should be confirmed in larger trials.

The strengths of our study include adequate randomization and the use of intention-to-treat analysis, both of which minimize the risk of bias. However, there are several limitations. First of all, the small sample size. Baseline characteristics were similar in the two groups of patients, and patients were randomized according to a computergenerated protocol with a single-blind design in order to minimize the risk of bias.

Second, clinical measures (i.e., number of bowel movements and duration of diarrhea) were used as outcomes. These measures alone are not considered optimal, indeed stool output is the main criterion defined by the World Health Organization to conclude that a drug is effective in acute diarrhea [22]. Since gelatin tannate was used as outpatient therapy, we could not assess the $24 \mathrm{~h}$ stool output, and we decided to evaluate clinical outcomes of efficacy. We evaluated patients at 24, 48 and $72 \mathrm{~h}$, and we made an additional visit at day 7 , in order to obtain accurate data, and we used a symptom diary for the entire duration of the study. Furthermore, we monitored common clinical symptoms such as fever, vomiting, abdominal pain, development of any other new symptoms and any hypersensitivity reaction like skin rashes to define potential adverse effects of intervention.

In contrast to other studies, we did not perform stool cultures at baseline [20, 23]. Nevertheless, ESPGHAN does not recommend performing stool cultures for acute gastroenteritis in primary healthcare, and we did not aim to evaluate the impact of gelatin tannate based on the different etiologies of diarrhea [2]. Finally, in our study design, patients were not hospitalized, the control group did not receive a placebo, and there was no direct monitoring of drug administration.

\section{Conclusions}

In conclusion, the results of this study suggest that gelatin tannate could be effective, well-tolerated, and safe in addition to oral rehydration therapy in infants and children with acute diarrhea. Further randomized, double-blind, placebo-controlled studies are needed to confirm its efficacy and safety and to evaluate its routine use in children with acute gastroenteritis.

\section{Compliance with Ethical Standards}

The study protocol was defined in accordance with the Declaration of Helsinki, and was approved by the ethical committee of each participating center (number of protocol 593/12). Written informed consent was obtained from parents of all children.

Conflict of interest Maurizio Mennini M.D., Carlo Tolone, M.D., Antonella Frassanito M.D., Fabio Midulla M.D. Ph.D., Salvatore Cucchiara M.D. Ph.D. and Marina Aloi M.D., Ph.D. declare that they have no conflict of interest and no financial relationships relevant to this article to disclose.

Funding source No external funding was received for this manuscript.

Contributors' statement Dr. Aloi and Dr Mennini conceptualized and designed the study, drafted the initial manuscript, and approved the final manuscript as submitted. Dr. Tolone and Dr. Mennini allocated and managed enrolled patients. Dr. Aloi and Dr. Frassanito designed the data collection instruments and carried out the initial analyses. Prof. Cucchiara and Prof. Midulla critically reviewed the manuscript and approved the final manuscript as submitted.

Availability of data and materials Data will not be shared, because of institutional policy. We will, however, be available to share them in the event of a specific request from a reviewer.

\section{References}

1. Elliott EJ. Acute gastroenteritis in children. BMJ. 2007;6(334):35-40. 
2. Guarino A, Ashkenazi S, Gendrel D, et al. European Society for Pediatric Gastroenterology, Hepatology, and Nutrition/European Society for Pediatric Infectious Diseases evidence-based guidelines for the management of acute gastroenteritis in children in Europe: update 2014. J Pediatr Gastroenterol Nutr. 2014;59:132-52.

3. Rahaman MM, Aziz KM, Patwari Y, et al. Diarrhoeal mortality in two Bangladeshi villages with and without community-based oral rehydration therapy. Lancet. 1979;20(2):809-12.

4. Pollard H, Moreau J, Ronco P, et al. Immunoautoradiographic localisation of enkephalinase (EC 3.4.24.11) in rat gastrointestinal tract. Neuropeptides. 1991;19:169-78.

5. Szajewska H, Skórka A, Ruszczyński M, et al. Meta-analysis: lactobacillus GG for treating acute gastroenteritis in childrenupdated analysis of randomised controlled trials. Aliment Pharmacol Ther. 2013;38:467-76.

6. Lehert P, Chéron G, Calatayud GA, et al. Racecadotril for childhood gastroenteritis: an individual patient data meta-analysis. Dig Liver Dis. 2011;43:707-13.

7. Das RR, Sankar J, Naik SS. Efficacy and safety of diosmectite in acute childhood diarrhoea: a meta-analysis. Arch Dis Child. 2015;100:704-12.

8. Farthing M. Novel targets for the pharmacotherapy of diarrhoea: a view for the millennium. J Gastroenterol Hepatol. 2000;15:38-45.

9. Yoshida T, Amakura Y, Yoshimura M. Structural features and biological properties of ellagitannins in some plant families of the order myrtales. Int J Mol Sci. 2010;11:79-106.

10. Esteban Carretero J, Durbán Reguera F, López-Argüeta Alvarez $\mathrm{S}$, et al. A comparative analysis of response to ORS (oral rehydration solution) vs ORS + gelatin tannate in two cohorts of pediatric patients with acute diarrhea. Rev Esp Enferm Dig. 2009;101:41-8

11. Scaldaferri F, Lopetuso LR, Petito V, et al. Gelatin tannate ameliorates acute colitis in mice by reinforcing mucus layer and modulating gut microbiota composition: emerging role for 'gut barrier protectors' in IBD? United European Gastroenterol J. 2014;2:113-22.
12. Lopetuso LR, Scaldaferri F, Bruno G, et al. The therapeutic management of gut barrier leaking: the emerging role for mucosal barrier protectors. Eur Rev Med Pharmacol Sci. 2015;19:1068-76.

13. Yoshida T, Amakura Y, Yoshimura M. Structural features and biological properties of ellagitannins in some plant families of the order myrtales. Int J Mol Sci. 2010;11(1):79-106.

14. Allegrini A, Costantini M. Gelatine tannate for the treatment of acute diarrhoea in adults. J Gastroint Dig Syst. 2012;2:3.

15. Ruszczyński M, Urbańska M, Szajewska H. Gelatin tannate for treating acute gastroenteritis: a systematic review. Ann Gastroenterol. 2014;27:121-4.

16. Chumpitazi BP, Lane MM, Czyzewski DI, et al. Creation and initial evaluation of a Stool Form Scale for children. J Pediatr. 2010;157:594-7.

17. Lane MM, Czyzewski DI, Chumpitazi BP, et al. Reliability and validity of a modified Bristol Stool Form Scale for children. J Pediatr. 2011;159(437-441):e1.

18. Friedman JN, Goldman RD, Srivastava R, et al. Development of a clinical de-hydration scale for use in children between 1 and 36 months of age. J Pediatr. 2004;145:201-7.

19. Ashok PK, Upadhyaya K. Tannins are astringent. J Pharmacognosy Phytochem. 2012;1:45-50.

20. Salazar-Lindo E, Santiesteban-Ponce J, Chea-Woo E, et al. Racecadotril in the treatment of acute watery diarrhea in children. N Engl Med J. 2000;343:463-7.

21. Strand TA, Sharma PR, Gjessing HK, et al. Risk factors for extended duration of acute diarrhea in young children. PLoS One. 2012;7:e36436.

22. United Nations Children's Fund and World Health Organization, 'WHO/UNICEF Joint Statement: Clinical management of acute diarrhoea', UNICEF, New York, 2004. http://www.afro.who.int/ cah/documents/intervention/acute_diarrhoea_joint_statement.pdf. Accessed June 2009.

23. Cezard J, Duhamel J, Meyer M, et al. Efficacy and tolerability of Racecadotril in acute diarrhea in children. Gastroenterology. 2001;120(799-805):13. 